\title{
Quadrilateral space syndrome
}

\author{
Omar Zurkiya \\ Division of Interventional Radiology, Massachusetts General Hospital/Harvard Medical School, Boston, MA, USA \\ Correspondence to: Omar Zurkiya, MD, PhD. Division of Interventional Radiology, Massachusetts General Hospital/Harvard Medical School, Boston, \\ MA 02114, USA. Email: ozurkiya@mgh.harvard.edu.
}

\begin{abstract}
The quadrilateral space is a confined area through which the axillary nerve and posterior circumflex humeral artery (PCHA) travel in the shoulder. Both structures are susceptible to impingement and compression as they travel though this space resulting in a constellation of symptoms known as quadrilateral space syndrome (QSS). Patients may experience paresthesias, loss of motor function, pain and vascular complications. Individuals who perform repetitive overhead arm movements such as elite athletes are at greater risk of developing QSS. The diagnosis can be difficult, but in the setting of clinical suspicion, physical exam and imaging studies can provide specific findings. On MRI, patients may have atrophy of the deltoid or teres minor muscles and angiography may show aneurysm or vascular occlusion of the PCHA. Treatment is initially conservative, with physiotherapy. Surgical decompression is effective in patients with severe or progressive symptoms. Causes of external compression such as fibrous bands, scarring, or other space occupying lesion may be addressed at that time. Neurolysis and aneurysm resection may also be performed. In some cases, emboli from the PCHA can cause ischemia in the involved upper extremity resulting in an acute presentation. Catheter directed therapy such as thrombolysis or thrombectomy may performed emergently in these cases. Though rare, in patients presenting with arm weakness, paresthesia, pain and/or arterial thrombosis in the arm, QSS is an important entity to consider.
\end{abstract}

Keywords: Quadrilateral space; quadrilateral space syndrome (QSS); compression syndromes

Submitted Jan 29, 2020. Accepted for publication Jun 04, 2020.

doi: $10.21037 /$ cdt-20-147

View this article at: http://dx.doi.org/10.21037/cdt-20-147

\section{Introduction}

Compressive disorders can arise when structures such as arteries, veins, and/or nerves pass through a confined anatomical space. Such is the case for the axillary nerve and posterior circumflex artery as they pass through the "quadrilateral space". The anatomical boundaries of this space, sometimes called the quadrangular space, are defined by the teres minor superiorly, the teres major inferiorly, the long head of the triceps brachii medially, and humeral neck laterally (Figure 1).

When the axillary nerve and/or the posterior circumflex humeral artery (PCHA) are compressed as they pass through the quadrilateral space, the constellation of symptoms that may arise is known as quadrilateral space syndrome (QSS). This term was introduced by Cahill and Palmer (1) in 1983 with reference to a series of patients who fared poorly after thoracic outlet surgery. Many of these patients had obstruction of the posterior circumflex artery leading to the recognition of QSS as a separate entity. Patients are typically young adults, with the dominant extremity involved. Symptoms include the slow onset of pain and paresthesia with flexion, abduction and external rotation increasing the symptoms. Aggravating factors may include growth of a mass, injury to the adjacent structures, or simply muscular hypertrophy, as may be seen in elite athletes. Non-focal shoulder pain, paresthesias, and tenderness to palpation in the region of the quadrilateral space are classic findings $(2,3)$. Frequently, these symptoms interfere with the patient's activities of daily living resulting in a notable effect on quality of life.

In the general population, QSS is a rare entity. In one study, Cothran and Helms reported only 19 out 2,436 

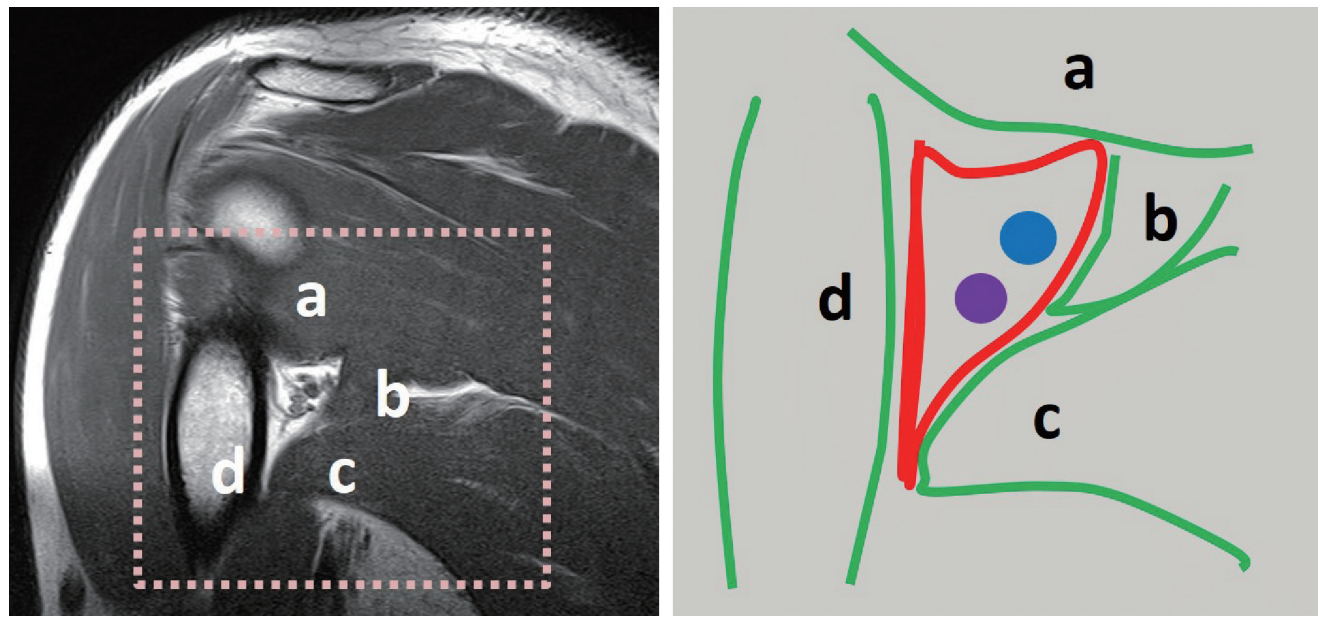

Figure $1 \mathrm{MRI}$ image (left) and pictorial representation of the area enclosed by dotted square (right) showing the structures creating the quadrilateral space, (a) teres minor, (b) long head of the triceps, (c) teres major, (d) surgical neck of the humerus; (blue circle) PCHA, (purple circle) axillary nerve. PCHA, posterior circumflex humeral artery.

receiving shoulder MRI had findings of QSS such as focal atrophy of the teres minor (4). Because of the rarity, many clinicians do not include QSS in their initial consideration of shoulder pathology. Indeed, of the 19 patients found to have QSS in the study, 13 were presumed to have rotator cuff tears.

The prevalence of QSS is likely much higher in elite athletes, particularly those with repetitive overhead arm movement. PHCA aneurysms were found by ultrasound (US) to be present in $4.6 \%(13 / 280)$ of elite volleyball players (5). Intravascular thrombus was identified in three cases. Ten of the aneurysms (77\%) were found within $1 \mathrm{~cm}$ of the origin of the PCHA from the axillary artery. Baseball players, quarterbacks, military personnel, and racquetball players have also been reported to have similar findings (6).

It is therefore felt that PCHA aneurysm formation is secondary to repetitive trauma from abduction and external rotation of the arm $(6,7)$. Some patients may develop further thrombosis of the PCHA. In these instances, patients may present acutely with distal embolization causing peripheral ischemia. Associated symptoms include pain, absent pulses and pallor requiring urgent thrombolysis and/or thrombectomy.

\section{Diagnosis}

Diagnosis of QSS can be difficult clinically as patients often present with vague, non-focal symptoms. QSS is typically characterized by a slow-onset or intermittent non-localized shoulder pain. Prior history of trauma or visible hypertrophy in the shoulder may raise suspicion. Worsening symptoms with abduction and external rotation may be present (1). Deltoid and teres minor weakness may be masked by recruitment of adjacent muscle groups to compensate for the lost function. On physical exam, there is frequently discrete point tenderness posteriorly in the quadrilateral space. Palpation of the radial pulse may find decreased flow during prolonged abduction though this finding overlaps with arterial thoracic outlet syndrome (aTOS), a separate compressive syndrome. There may be paresthesia of the posterior or lateral aspect of the arm and shoulder with preserved sensation to light touch. Throwing athletes may describe pain and fatigue with the arm in overhead position.

Shoulder pain and weakness are common presenting symptoms and common entities like rotator cuff pathology and degenerative changes may be present in patients who have QSS. It is important for providers to be aware that various common pathologies such as presence of rotator cuff tendinopathy do not mean QSS is excluded. The clinical exam should be directed towards identifying the specific muscle or muscles involved. A finding of atrophy of deltoid or teres minor should raise suspicion of QSS even in patients with known tendinopathy or shoulder or acromioclavicular joint pathologies.

In addition to the common shoulder pathologies mentioned above, the differential diagnosis of QSS includes cervical radiculopathy causing referred pain, brachial 

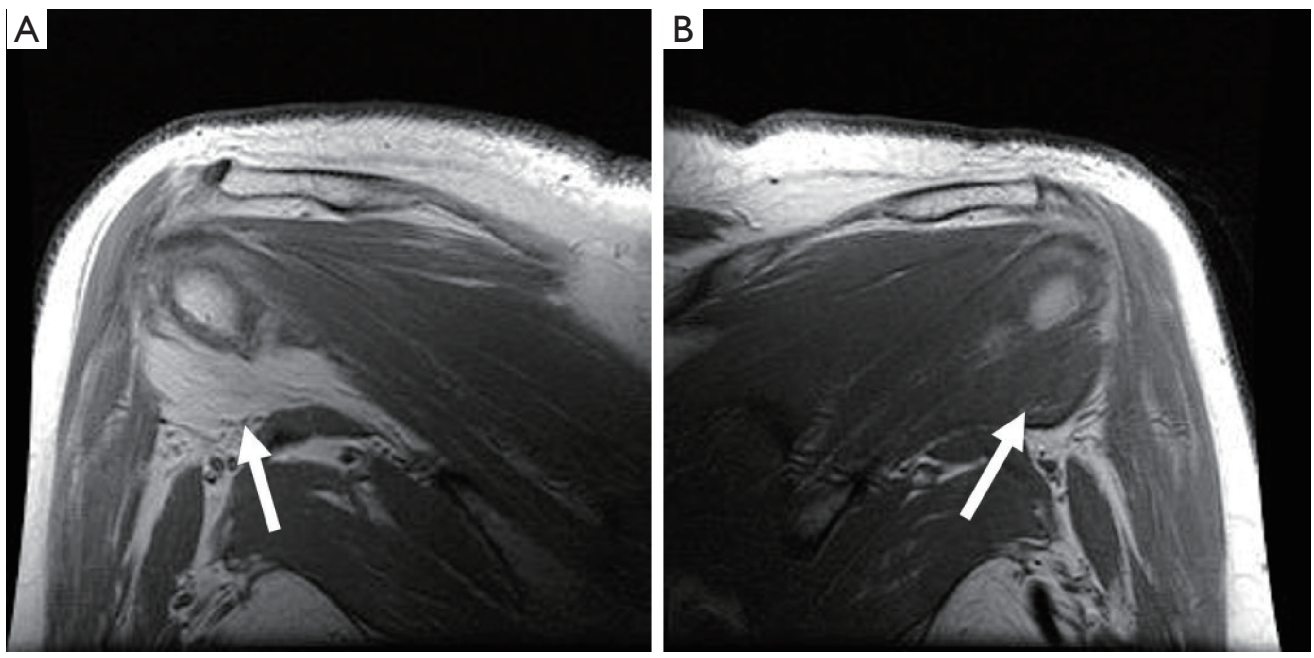

Figure 2 MRI images of the right (A) and left (B) shoulders from the same patient with right QSS. In panel (A), the arrow points to fatty atrophy of the teres minor. In panel (B), the normal teres minor is seen (arrow). QSS, quadrilateral space syndrome.

neuritis, suprascapular nerve injury and TOS.

Nerve conduction studies may show axillary neuropathy and electromyography can show active denervation potential from the deltoid and teres minor. Electromyograms may also rule out other neurologic entities such as pain from the cervical spine or brachial plexus.

US, if used, may show altered architecture of involved muscle $(8,9)$. Lesions of the axillary nerve or other space occupying lesions could be identified. The PCHA may be identified with doppler US to demonstrate occlusion or aneurysm formation. One study of US assessment of the PCHA in 280 elite volleyball players (5) found that the PCHA could be identified in $100 \%$ of cases. PCHA aneurysms were found in $4.6 \%(13 / 280)$ of patients originating from the axillary artery. The aneurysms were characterized as fusiform with 10 occurring within $1 \mathrm{~cm}$ of the origin of the axillary artery. Intravascular thrombus could be identified in three cases. Aneurysm size could be measured $(5.9 \pm 1.7 \mathrm{~mm}$ in males and $5.2 \pm 0.2 \mathrm{~mm}$ in females). US remains highly operator dependent and cannot identify fibrous bands which commonly cause QSS. However, this study suggests its potential use as a screening tool in elite athletes.

Plain films could demonstrate osseous etiologies such as osteochondromas or post-traumatic remodeling impinging on the quadrilateral space. These are limited in providing soft tissue diagnosis however may be helpful in identifying other causes of shoulder pain such degenerative conditions.

Computed tomography (CT) can be used to identify lesions that may impinge on the quadrilateral space and is particularly useful in identifying osseous abnormalities from etiologies such as trauma or degenerative change. CT angiography (CTA) is a non-invasive vascular imaging modality in which images are obtained in arterial and venous phases after intravenous iodinated contrast injection. This can be used to identify PCHA abnormalities including aneurysms and thrombosis as well as distal thrombi within the arm (3).

MRI has traditionally been used to demonstrate fatty atrophy of the teres minor or deltoid muscles $(4,10)$. Typical findings on $\mathrm{T} 1$ weighted sequences include atrophy and fatty infiltration (Figure 2) but space occupying lesions such as neuromas, tumors, hypertrophied adjacent muscles or vascular aneurysms may be seen. T2-weighted sequences, typically fast spin-echo with fat suppression are used to detect abnormalities such as paralabral cysts. Diffuse increased T2 signal in the teres minor may suggest neurogenic edema consistent with QSS. MR angiography (MRA) can be used similarly to CTA to identify vascular abnormalities in the PCHA and distally in the arm. MRI has also been described as a possible modality to evaluate for axillary nerve compression, particularly at high fields such as 3 Tesla (11). Contributing shoulder pathologies such as rotator cuff or labral pathology can be identified by MRI or MR arthrography. In the case of MR arthrography, contrast is injected directly in the shoulder joint with fluoroscopic guidance followed by MR imaging, making it more sensitive for tears and paralabral pathology. Cahill and 

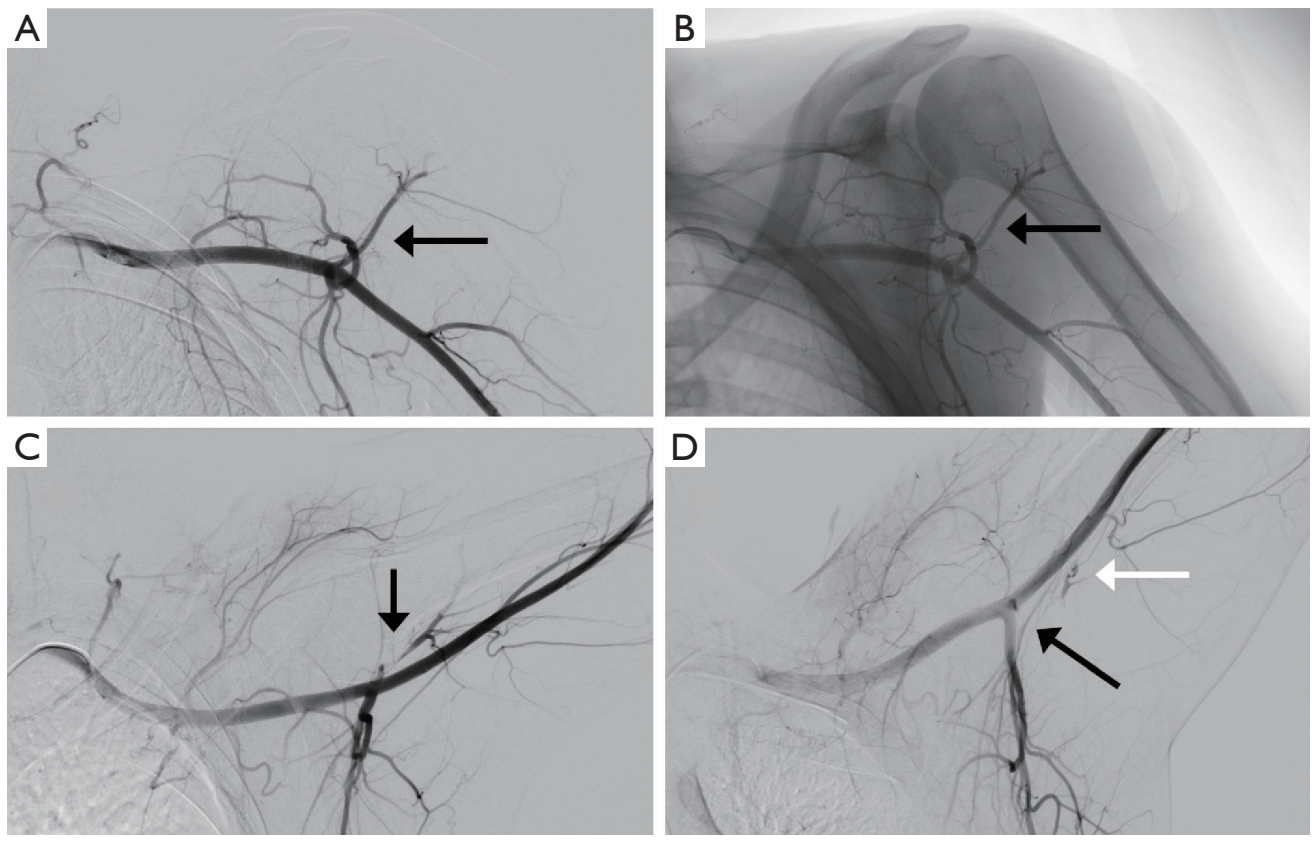

Figure 3 Angiography in a patient with QSS. (A) DSA in neutral position showing patent PCHA (arrow); (B) non-subtracted image from the same injection as panel a, to highlight bony landmarks; (C) DSA obtained in slightly elevated position shows narrowing of the PCHA (arrow); (D) DSA obtained in overhead position showing complete occlusion of the PCHA (black arrow) with retrograde filling of a portion of the PCHA (white arrow). QSS, quadrilateral space syndrome; DSA, digital subtraction angiography; PCHA, posterior circumflex humeral artery.

Palmer suggested the use of angiography as a diagnostic tool (1). Fluoroscopic and digital subtraction angiographic images are obtained after intra-arterial contrast injection into the subclavian or axillary artery. Images are obtained in both the neutral and abducted/externally rotated positions. The PCHA may be attenuated or entirely occluded with findings more pronounced in the abducted position (Figure 3). For non-acute patients suspected of QSS, diagnostic angiography could also identify chronic arterial changes such as aneurysm formation, dissection or partially occlusive thrombus allowing for intervention prior to the appearance of emboli or acute occlusion. In the acute stages, peripheral ischemia from emboli originating in the PCHA may be present with angiography showing complete arterial occlusion or aneurysm formation in the PCHA. In these patients, angiography is not only diagnostic, but allows for immediate intervention such as with thrombolysis.

\section{Treatment}

Initial treatment is generally conservative with targeted symptomatic care and lifestyle changes. Activity is modified to avoid abduction and external rotation of the affected limb. Physiotherapy is typically the first line intervention for QSS including strengthening of the rotator cuff muscles, glenohumeral joint mobilization, and scapular stabilization exercises (3). Soft tissue massage to the quadrilateral space may also be implemented including targeted friction and active release massage. These conservative approaches should be used for a minimum of 6 months before more invasive interventions are considered. Nonsteroidal antiinflammatory drugs (NSAIDs) may be prescribed.

Lidocaine block, with or without fluoroscopic guidance can be done to assess whether the quadrilateral space is the source of pain. Steroid injection may similarly be performed for longer term therapeutic benefit $(12,13)$.

In patients who presently with acute symptoms such as discoloration, pale or cool hand or digits, absent or diminished Doppler signals at the wrist, anticoagulation should be initiated and endovascular intervention may be performed. An arch angiogram with upper extremity runoff followed by catheter directed thrombolysis may be performed. After a period of up to 48 hours, thrombolysis may then be followed with therapeutic anticoagulation for 
several months (6).

If there is no response, or worsened symptoms, decompression surgery can be performed $(6,14)$. The quadrilateral space is exposed by making a longitudinal incision over the posterior shoulder and the deltoid is moved superolaterally to access the quadrilateral space between the teres minor and major. Excision of fibrous bands, scarring, or other space occupying lesion is performed. Neurolysis and aneurysm resection may also be necessary. In cases of thrombus originating from trauma to the PCHA, this artery may be ligated or occluded with endovascular techniques. Anticoagulation may be continued for a period of 3 to 6 months after surgery.

Arthroscopic debridement may be performed instead of, or after decompression to address intra-articular pathology such as labral tears or paralabral cysts that could be causing compression of the quadrilateral space (3).

Post-operatively, early physical therapy is implemented to prevent new adhesion formation. Hyperextension, abduction and external rotation should be initially avoided for at least 4 weeks, however pendulum exercises can be safely started in the immediate post-operative period. Athletes may continue to sport-specific exercise no earlier than 6 weeks from surgery.

\section{Conclusions}

QSS remains a rare entity that can be difficult to diagnose. Acute events such as PCHA thrombosis with or without aneurysm formation can result in distal embolization and ischemia that point directly to QSS. Space occupying lesions in the quadrilateral space may be identified, but are difficult to identify without specific diagnostic direction from clinical suspicion. Elite athletes may present with QSS however shoulder injuries and TOS are typically the first diagnostic considerations. As a result, QSS is frequently a diagnosis of exclusion. Point tenderness in the quadrilateral space or diagnostic imaging showing deltoid and/or teres minor atrophy are specific findings pointing to QSS. Treatment is generally conservative at first however surgical decompression has been shown to be efficacious in the patients who do not respond or progress on non-surgical therapies.

\section{Acknowledgments}

Funding: None.

\section{Footnote}

Provenance and Peer Review: This article was commissioned by the Guest Editors (Sanjeeva Kalva and Marcin Kolber) for the series "Compressive Vascular Syndromes" published in Cardiovascular Diagnosis and Therapy. The article has undergone external peer review.

Conflicts of Interest: The author has completed the ICMJE uniform disclosure form (available at http://dx.doi. org/10.21037/cdt-20-147). The series "Compressive Vascular Syndromes" was commissioned by the editorial office without any funding or sponsorship. The author has no other conflicts of interest to declare.

Ethical Statement: The author is accountable for all aspects of the work in ensuring that questions related to the accuracy or integrity of any part of the work are appropriately investigated and resolved.

Open Access Statement: This is an Open Access article distributed in accordance with the Creative Commons Attribution-NonCommercial-NoDerivs 4.0 International License (CC BY-NC-ND 4.0), which permits the noncommercial replication and distribution of the article with the strict proviso that no changes or edits are made and the original work is properly cited (including links to both the formal publication through the relevant DOI and the license). See: https://creativecommons.org/licenses/by-nc-nd/4.0/.

\section{References}

1. Cahill BR, Palmer RE. Quadrilateral space syndrome. J Hand Surg Am 1983;8:65-9.

2. Hangge PT, Breen I, Albadawi H, et al. Quadrilateral space syndrome: diagnosis and clinical management. J Clin Med 2018;7:86.

3. Flynn LS, Wright TW, King JJ. Quadrilateral space syndrome: a review. J Shoulder Elbow Surg 2018;27:950-6.

4. Cothran RL Jr, Helms C. Quadrilateral space syndrome: incidence of imaging findings in a population referred for MRI of the shoulder. AJR Am J Roentgenol 2005;184:989-92.

5. van de Pol D, Maas M, Terpstra A, et al. Ultrasound assessment of the posterior circumflex humeral artery in elite volleyball players: Aneurysm prevalence, anatomy, branching pattern and vessel characteristics. Eur Radiol 
2017;27:889-98.

6. Brown SA, Doolittle DA, Bohanon CJ, et al. Quadrilateral space syndrome: the Mayo Clinic experience with a new classification system and case series. Mayo Clin Proc 2015;90:382-94.

7. Menon D, Onida S, Davies AH. Overview of arterial pathology related to repetitive trauma in athletes. J Vasc Surg 2019;70:641-50.

8. Chen H, Onishi K, Zhao X, et al. Neuromuscular ultrasound application to the electrodiagnostic evaluation of quadrilateral space syndrome. PM R 2014;6:845-8.

9. Brestas PS, Tsouroulas M, Nikolakopoulou Z, et al. Ultrasound findings of teres minor denervation in suspected quadrilateral space syndrome. J Clin Ultrasound 2006;34:343-7.

10. Helms CA. The impact of MR imaging in sports medicine.
Radiology 2002;224:631-5.

11. Chhabra A, Madhuranthakam AJ, Andreisek G. Magnetic resonance neurography: current perspectives and literature review. Eur Radiol 2018;28:698-707.

12. Bourget-Murray J, Davison E, Frizzell B, et al.

Fluoroscopic-guided quadrilateral space block for the treatment of quadrilateral space syndrome - a case report. J Clin Orthop Trauma 2019;10:655-8.

13. Chang PS, Jacobson N, Chang KU. Quadrilateral space syndrome treated with ultrasound-guided corticosteroid injection: a case of isolated teres minor atrophy and review of the literature. S D Med 2017;70:444-7.

14. McAdams TR, Dillingham MF. Surgical decompression of the quadrilateral space in overhead athletes. Am J Sports Med 2008;36:528-32.
Cite this article as: Zurkiya O. Quadrilateral space syndrome. Cardiovasc Diagn Ther 2021;11(5):1112-1117. doi: 10.21037/ cdt-20-147 